\title{
A Polarized Target Measurement of the Electric Form Factor of the Neutron at JLab
}

\author{
N. Savvinov (on behalf of JLab E93-026 collaboration) \\ University of Maryland, College Park, USA
}

Received on 10 September, 2003

\begin{abstract}
The experiment E93-026 at the Thomas Jefferson National Accelerator Facility (JLab) determined the electric form factor of the neutron $G_{E}^{n}$ through quasielastic $\vec{d}\left(\vec{e}, e^{\prime} n\right) p$ scattering using a longitudinally polarized electron beam and a frozen polarized ${ }^{15} N D_{3}$ target. The knocked out neutrons were detected in a segmented plastic scintillator detector in coincidence with the scattered electrons. The $G_{E}^{n}$ was extracted by comparing the experimental beam-target asymmetry with full theoretical calculations based on different values of $G_{E}^{n}$. Preliminary results of the Fall 2001 run are reported.
\end{abstract}

\section{Introduction}

The electric form factor of the neutron $G_{E}^{n}$ is of fundamental importance for both nuclear and particle physics. It provides a unique testing ground for QCD models in the intermediate energy regime (where perturbative methods cannot be applied). Also, $G_{E}^{n}$ is an important input for various experiments studying the strange content of the nucleon, structure functions of few-body systems, charge radii of nuclei, etc.

Until the last decade, our knowledge of $G_{E}^{n}$ was very scarce. The best determination of $G_{E}^{n}$ from elastic electrondeuteron scattering provided data only up to $1(\mathrm{GeV} / \mathrm{c})^{2}$ and suffered from large (30-40\%) dependence on the choice of the $N-N$ interaction potential. The situation has significantly improved since the advent of polarized methods in early 1990s, made possible by technological progress in high duty factor accelerators, polarized targets and recoil polarimeters. However, the uncertainties are still large and the explored $Q^{2}$ range is still low. A massive experimental program currently in progress at JLab and other nuclear facilities around the world is aiming to further improve our knowledge of $G_{E}^{n}$.

In the experiment presented here a longitudinally polarized electron beam was scattered off a polarized deuterated ammonia target. The helicity induced asymmetry (given by the ratio of helicity-flip and helicity independent parts of the scattering cross section) is sensitive to $G_{E}^{n}$. For the ideal case of a free neutron target with the polarization perpendicular to the momentum transfer and lying in the scattering plane, the asymmetry $A_{e n}$ is:

$$
A_{e n}=\frac{-2 \sqrt{\tau(1+\tau)} G_{M}^{n} G_{E}^{n}}{\left(G_{E}^{n}\right)^{2}+\tau\left(1+2(1+\tau) \tan ^{2}\left(\theta_{e} / 2\right)\right)\left(G_{M}^{n}\right)^{2}},
$$

where $G_{M}^{n}$ is the magnetic form factor of the neutron, $\tau=$ $\frac{Q^{2}}{4 M^{2}}, M$ is the neutron mass, and $\theta_{e}$ is the electron scattering angle.

Since neutrons in deuterium are not free, the measured electron-deuteron asymmetry $A_{e d}^{V}$ differs from $A_{e n}$ due to reaction mechanisms such as final state interactions and meson exchange currents. These reaction mechanisms were taken into account in theoretical calculations used for extraction of $G_{E}^{n}[1]$.

\section{Experimental setup}

The experiment E93-026 was conducted in Hall C of JLab in 1998 and 2001. The measurements were taken at two points, $Q^{2}=0.5(\mathrm{GeV} / \mathrm{c})^{2}$ and $1.0(\mathrm{GeV} / \mathrm{c})^{2}$ (henceforth the units of $Q^{2}$ are assumed to be $\left.(\mathrm{GeV} / \mathrm{c})^{2}\right)$.

The beam polarization was measured using a Moeller polarimeter. The average beam polarization was $78.2 \%$ (71.8\%) for $Q^{2}=0.5\left(Q^{2}=1.0\right)$. The beam current was limited to $100 \mathrm{nA}$ to avoid excessive thermal and radiation damage to the target polarization. A system of raster magnets was used to distribute the beam uniformly over the face of the target. The deflection of incident electrons by the target magnetic field was compensated by two chicane magnets.

The solid polarized target [2] was developed by University of Virginia. The basic components of the polarized target include a superconducting magnet operated at 5 Tesla, a ${ }^{4} \mathrm{He}$ evaporation refrigerator, a pumping system, a high power microwave tube operating at frequencies around 140 $\mathrm{GHz}$ and an NMR system for measuring the target polarization. The target material was polarized using the principle of dynamic nuclear polarization. Target polarization was determined by measuring the impedance change of the series resonant LCR circuit due to the nuclear magnetic moment. The conversion constants between the area of the NMR signal and the target polarization were obtained by a series of thermal equilibrium measurements. The target polarization typically varied between $15 \%$ and $35 \%$ and averaged to $24.1 \%(23.8 \%)$ for $Q^{2}=0.5\left(Q^{2}=1.0\right)$.

After interaction in the target material, the scattered electrons were detected in the High Momentum Spectrome- 
ter (HMS) of Hall C. Recoil nucleons were detected in the neutron detector which consisted of six planes of thick scintillators and two planes of thin scintillators. The latter were used for particle identification. The detector was set along the direction of the three-momentum transfer and was enclosed in a concrete hut open towards the target. The neutron vertical position was determined by the segmentation of the detector while the horizontal position was determined from the time difference of the phototubes.

\section{Analysis and results}

The electrons in the HMS were reconstructed using the standard HMS reconstruction code extended for the effects of the beam raster and the target magnetic field. On the neutron detector side a custom tracking algorithm was developed for proper particle identification. Neutrons were defined as events with no hits in the paddles along the track to the target, within a narrow coincidence time window and within a $100 \mathrm{MeV}$ range of invariant mass around the nucleon mass. A number of other cuts was applied to optimize the dilution factor and keep the nuclear corrections under control.

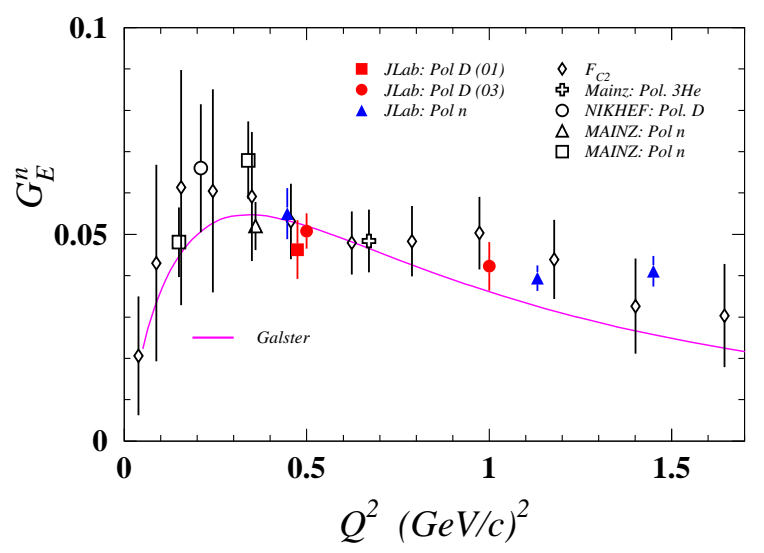

Figure 1. Results of 1998 (filled square) and 2001 (filled circles) runs of JLab E93-026 compared to other recent polarized data ( filled triangles [3], open diamonds [4], open cross [5], open circle [6], open triangle [7], open squares [8]).

After event reconstruction and event selection, the raw asymmetry $\epsilon$ was calculated from charge and deadtime normalized yields for the two helicity states, $N^{+}$and $N^{-}$:

$$
\epsilon=\frac{N^{+}-N^{-}}{N^{+}+N^{-}}=P_{B} P_{T} f A_{e d}^{V},
$$

where $f$ is the dilution factor due to scattering from unpolarized materials and $P_{B}$ and $P_{T}$ are the beam and target polarizations. The dilution factor was calculated using Monte Carlo simulations. A number of corrections was applied to $A_{e d}^{V}$. The leading correction was the one due to the charge exchange in the lead shielding: $-3.8 \pm 1.1 \%(-3.3 \pm 3.0 \%)$ for $Q^{2}=0.5\left(Q^{2}=1.0\right)$. The correction for the accidental background was $1.9 \pm 0.1 \%(0.5 \pm 0.2 \%)$ and the radiative effects were found to be small: $0.9 \pm 0.5 \%(0.6 \pm 0.5 \%)$. The $G_{E}^{n}$ was extracted by comparing the corrected experimental asymmetry to the theoretical asymmetry averaged over the experimental acceptance under different assumptions about the size of the $G_{E}^{n}$. The extracted value was corrected for the deviation of the magnetic form factor $G_{M}^{n}$ from the dipole parametrization [9] used in the theoretical calculations. The preliminary results are:

$$
\begin{aligned}
& G_{E}^{n}\left(Q^{2}=0.5\right)=0.0526 \pm 0.0033 \pm 0.0026 \\
& G_{E}^{n}\left(Q^{2}=1.0\right)=0.0454 \pm 0.0054 \pm 0.0037
\end{aligned}
$$

The leading contributions to the systematic error for $Q^{2}=$ $0.5\left(Q^{2}=1.0\right)$ are the dilution factor $3.4 \%(3.0 \%)$, the target polarization $2.9 \%(4.6 \%)$, the beam polarization $1.1 \%(3.3 \%)$ and the uncertainty in the central kinematic values $1.2 \%(3.4 \%)$.

\section{Summary}

The experiment E93-038 was successfully performed at the $\mathrm{JLab}$ in 2002. The experiment measured the neutron charge form factor at $Q^{2}=0.5$ and $1.0(\mathrm{GeV} / \mathrm{c})^{2}$. Preliminary results are in agreement with other recent polarized measurements.

\section{References}

[1] H. Arenhövel, et al., Z. Phys. A 331, 123 (1988).

[2] D. Crabb, et al., Nucl. Instr. Meth. A 356, 9 (1995).

[3] R. Madey, et al., preprint nucl-ex/0308007, submitted to Phys. Rev. Lett.

[4] I. Sick, et al., Phys. Rev. C 64, 041002 (2001).

[5] J. Bermuth, et al., preprint nucl-ex/0303015, submitted to Elsevier Science.

[6] I. Passchier, et al., Phys. Rev. Lett. 82, 4988 (1999).

[7] J. Golak, et al., Phys. Rev. C 63, 034006 (2001).

[8] C. Herberg, et al., Eur. Phys. J. A 5, 131 (1999).

[9] G. Kubon, et al., Phys. Lett. B 524, 26 (2002). 